\title{
Performance analysis of low-complexity welch power spectral density for automatic frequency analyser
}

\author{
Teh Yi Jun ${ }^{1}$, Asral Bahari Jambek ${ }^{2}$, Uda Hashim³ \\ ${ }^{1,2}$ School of Microelectronic Engineering, Universiti Malaysia Perlis, Perlis, Malaysia \\ ${ }^{3}$ Insituite of Nano Electronic Engineering, Universiti Malaysia Perlis, Perlis, Malaysia
}

\begin{tabular}{|c|c|}
\hline Article Info & ABSTRACT \\
\hline Article history: & The aim of this paper is to investigate the performance of the Low \\
\hline Received Oct 24, 2018 & $\begin{array}{l}\text { Complexity Welch Power Spectral Density Computation (PSDC). This } \\
\text { algorithm is an improvement from Welch PSDC method to reduce the }\end{array}$ \\
\hline Revised Nov 30, 2018 & computational complexity of the method. The effect of the sampling rate and \\
\hline Accepted Dec 21, 2018 & $\begin{array}{l}\text { the input frequency toward to accuracy of frequency detection is being } \\
\text { evaluated. From the experiment results, sampling rate nearest to the twice of }\end{array}$ \\
\hline Keywords: & ability of the algorithm to perform complex signal also has been investigated. \\
\hline
\end{tabular}

Frequency analyser

Frequency estimation

Power spectral density

\section{Corresponding Author:}

Teh Yi Jun,

School of Microelectronic Engineering,

University Malaysia Perlis,

Pauh Putra Campus, 02600 Pauh, Perlis, Malaysia.

Email: kelvinteh90@gmail.com

\section{INTRODUCTION}

Frequency based sensor have grown rapidly in popularity. Hence, the requirement of frequency analysis also highly increases. In the frequency analysis, the common process is the spectral density calculation. There are many methods of spectral density calculation been proposed, namely as Robust Spectral Density Estimation (RSDE), Independent Component Analysis (ICA), Multitaper Power Spectral Density Estimation (MPSDE), Low-complexity Welch Power Spectral Density (LCWPSD), B-Spline Windows Power Spectral Density Estimation and etc. [1-13]. Among the method listed, LCWPSD is the most suitable for biosensor processing. Hence, it has been proposed to apply in biosensor application. Optimum parameter are required to enhance the performance of LCWPSD. Next sections are going to discuss the details for LCWPSD.

\section{LITERATURE REVIEW}

In this section, the propose method will be discussed in details on how the spectral density of the signal is detected. LCWPSDC [4] is a modified method from the Welch PSDC [14] in term to reduce the complexity of computational. Figure 1 illustrated the flow of the LCWPSDC. Input signal first will be segmented into them with the length of $\mathrm{N}$. Then, the segment with the length of $\mathrm{N}$ will be divided into two windows which length of N/2. After dividing into two windows, the FFT will be applied to each window, the product called N/2-FFT. The N/2-FFT from the window at the same segment will be merge again become NFFT. The mathematical derivation of FFTs process is present as below. 


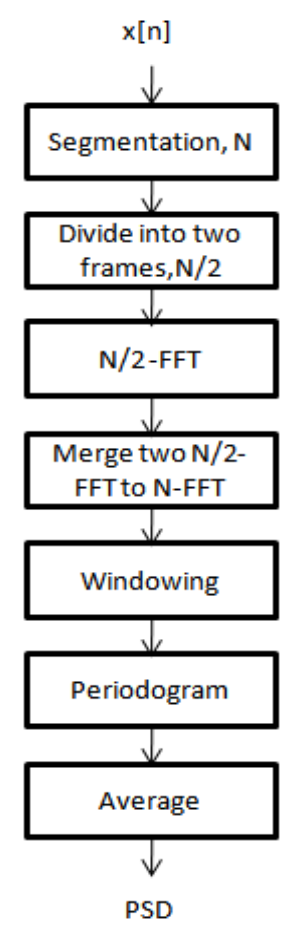

Figure 1. Flow of the low complexity Welch PSDC

The signal of every segment of the input signal as $x[n]$, where the $n=0, \ldots, N-1$ of length of $N$. Given the FFT of $x[n]$ as (1).

$$
X(k)=\sum_{n=0}^{N-1} x[n] e^{-j \frac{2 \pi}{N} n k}
$$

(1) was substituted with $k=s+2 u$ and $n=l+m M$, where $M=N / 2, s=\{0,1\}, u=0, \ldots$, $M-1, m=\{0,1\}$, and $l=0,1, \ldots, M-1$, we get ,

$$
\begin{aligned}
& X_{1}(k)=\sum_{n=0}^{M-1} x[n] e^{-j \frac{2 \pi}{M} n k} \\
& X_{2}(k)=\sum_{n=0}^{M-1} x[n+M] e^{-j \frac{2 \pi}{M} n k} \\
& X_{3}(k)=\sum_{n=0}^{M-1} x[n] e^{-j \frac{\pi n}{M}} e^{-j \frac{2 \pi}{M} n k} \\
& X_{3}(k)=\sum_{n=0}^{M-1} x[n] e^{-j \frac{\pi n}{M}} e^{-j \frac{2 \pi}{M} n k}
\end{aligned}
$$

To merge two N/2-FFT into N-FFT (6) and (7) are applied.

$$
\begin{aligned}
& X(2 u)=X_{1}(k)+X_{2}(k) \\
& X(2 u+1)=X_{3}(k)-X_{4}(k)
\end{aligned}
$$

After that the N-FFT will be applied with windowing process. In windowing process, Fractionaldelay (FD) finite impulse response (FIR) filter was applied. The coefficients of the FD FIR are determined using (8), where D is the delay with non-integer. In this case, delay is set as 0.5.

$$
h[n]=\left\{\begin{array}{lr}
\operatorname{sinc}\left(n-D-\frac{N}{2}+1\right), 0 \leq n \leq N-1 \\
0, & \text { otherwise }
\end{array}\right.
$$


After windowing process, the periodogram of each segment is determined. The last stage is to calculate the average of the periodogram from all the segments as the PSD of the signal. The LCWPSDC algorithm will simulate thru MATLAB.

\section{METHODOLOGY}

In Section 2, the LCWPSDC method was discussed. In this section, the experiment will evaluate the effect of the sampling rate and input frequency as well as the ability of algorithm to process complex signal. The experiment will be done using MATLAB simulation. Hence, three experiments will be conducted. The sinusoidal signals are selected as the input signal to perform the experiment. Figure 2 illustrated the original input signal of $20 \mathrm{~Hz}$ frequency.

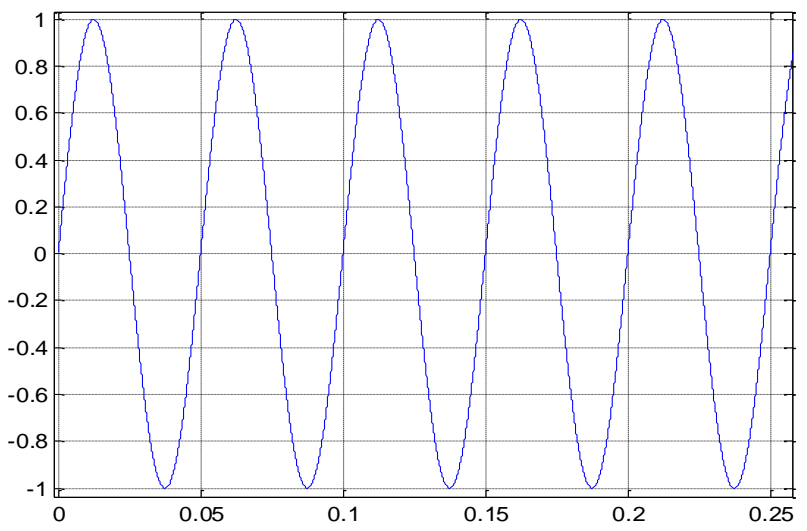

Figure 2. Original input signal of $20 \mathrm{~Hz}$ frequency

The first experiment is to determine the relationship between the sampling rate and the accuracy of the algorithm. This experiment used the $20 \mathrm{~Hz}$ of sinusoidal signal as the input signal. The sampling rate are varied from $50 \mathrm{~Hz}$ until $250 \mathrm{~Hz}$ with each increment of $50 \mathrm{~Hz}$. Figure 3 illustrated the sampled input signal of $20 \mathrm{~Hz}$ frequency using $150 \mathrm{~Hz}$ sampling rate. The second experiment is to determine the relationship between the input frequency and the accuracy of the algorithm. This experiment is set the sampling rate fix at $150 \mathrm{~Hz}$ while the input sinusoidal signal is varied from $10 \mathrm{~Hz}$ until $50 \mathrm{~Hz}$ with each increment $10 \mathrm{~Hz}$.

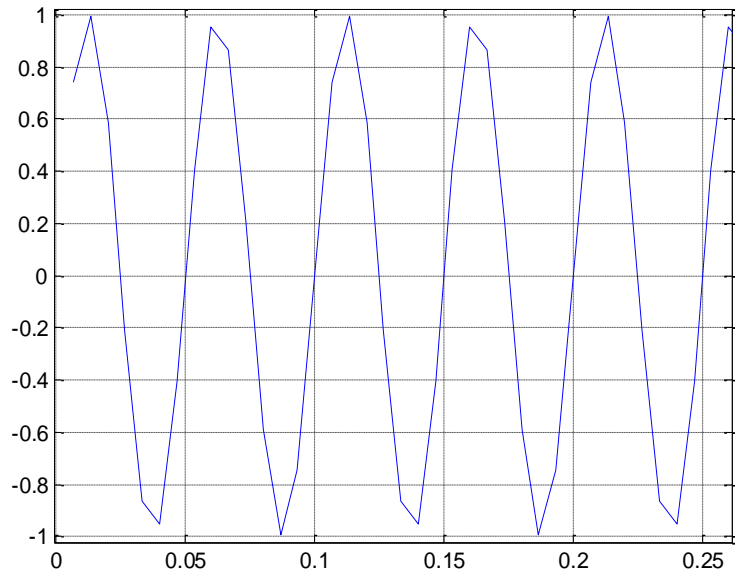

Figure 3. Sampled input signal of $20 \mathrm{~Hz}$ frequency using $150 \mathrm{~Hz}$ sampling rate

The third experiment is to study the ability of the algorithm to perform frequency detection from complex signal. This experiment is performed with complex input signal which is the summation of $10 \mathrm{~Hz}$, 
$30 \mathrm{~Hz}$ and $50 \mathrm{~Hz}$ sinusoidal signal. Figure 4 illustrated the sampled complex input signal of $10 \mathrm{~Hz}, 30 \mathrm{~Hz}$ and $50 \mathrm{~Hz}$ using $150 \mathrm{~Hz}$ sampling rate. Table 1 and Table 2 summarize the experimental setup.

Table 1. Experimental setup of first experiment

\begin{tabular}{cc}
\hline Input frequency & Sampling Rate \\
\hline 20 & 50 \\
20 & 100 \\
20 & 150 \\
20 & 200 \\
20 & 250 \\
\hline
\end{tabular}

Table 2. Experimental setup of second and third experiment

\begin{tabular}{cc}
\hline Input frequency & Sampling Rate \\
\hline 10 & 150 \\
20 & 150 \\
30 & 150 \\
40 & 150 \\
50 & 150 \\
Complex signal & 150 \\
\hline
\end{tabular}

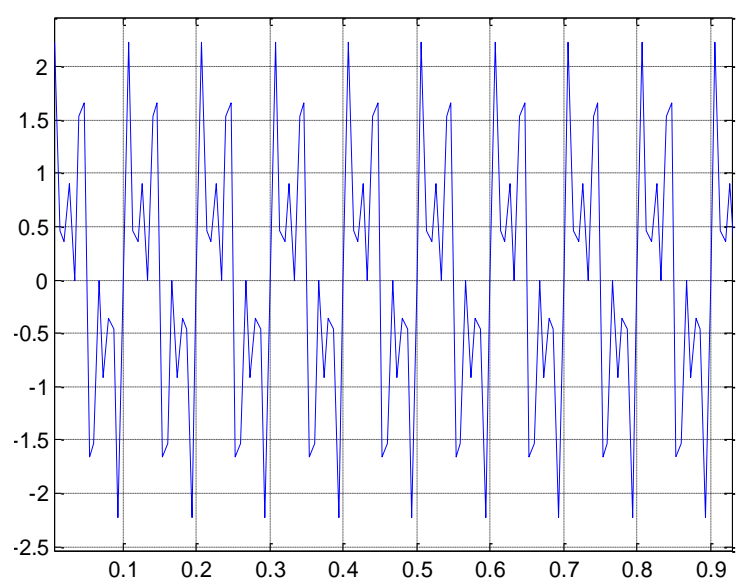

Figure 4. Sampled complex input signal of $10 \mathrm{~Hz}, 30 \mathrm{~Hz}$ and $50 \mathrm{~Hz}$ using $150 \mathrm{~Hz}$ sampling rate

\section{RESULTS AND DISCUSSIONS}

As the experiment discussed in Section 3, the results will be discussed in this section. All three experiments results will be discussed in detail. Figure 5 illustrated the spectral density detection that using algorithm LCWPSDC with $150 \mathrm{~Hz}$ sampling rate for input sinusoidal signal of $30 \mathrm{~Hz}$ frequency.

Table 3 shows the results of the first experiment to study the effect of sampling rate to the accuracy of the algorithm. From the results, sampling rate at $50 \mathrm{~Hz}$ is able to achieve $99.17 \%$ accuracy of frequency detection whereas sampling rate at $250 \mathrm{~Hz}$ obtained $96.24 \%$ accuracy of frequency detection. The results show that the accuracy are decreases as the sampling rate increases. This trend is observed because sampling rate will use as the scale during FFT and larger scale to measure the value will decrease the accuracy. Hence, the lowest sampling rate provides the highest accuracy and the accuracy is decreasing with the increasing of sampling rate.

Table 4 shows results for the second experiment to study the effect of input frequency to the accuracy of the algorithm. From the results, lowest input frequency with $10 \mathrm{~Hz}$ has $94.53 \%$ accuracy of frequency detection. The highest input frequency with $50 \mathrm{~Hz}$ has $98.93 \%$ accuracy of frequency detection. The results show that in accuracy is increasing while the input frequency is increasing. Base on the Nyquist rate, the lowest sampling rate must be at least twice of input frequency to avoid aliasing [15]. Hence, concluded from the first and second experiment, the sampling rate nearest to twice of input frequency will provide the highest accuracy.

Figure 6 illustrated the results of the third experiment to study the ability of algorithm on complex signal. From the results, it showed that the algorithm was able to detect the complex signals that consist of summation of $10 \mathrm{~Hz}, 30 \mathrm{~Hz}$ and $50 \mathrm{~Hz}$ with the frequencies of $10.55 \mathrm{~Hz}, 30.62 \mathrm{~Hz}$ and $50.54 \mathrm{~Hz}$. This results are compared to Table 4 where when each signal is detected individually for $10 \mathrm{~Hz}, 30 \mathrm{~Hz}$ and $50 \mathrm{~Hz}$ input frequencies are $10.55 \mathrm{~Hz}, 30.62 \mathrm{~Hz}$ and $50.54 \mathrm{~Hz}$ respectively. This shows that the algorithm detect the complex signal are same as the signal has been detected individually. Hence, it proves that the algorithm are able to perform on complex signal. 


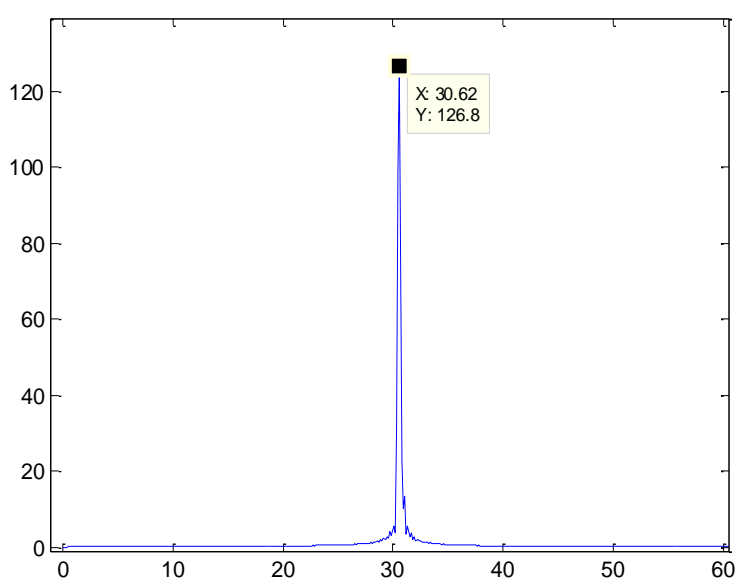

Figure 5. The results of $30 \mathrm{~Hz}$ sinusoidal signal as the input signal using $150 \mathrm{~Hz}$ sampling rate

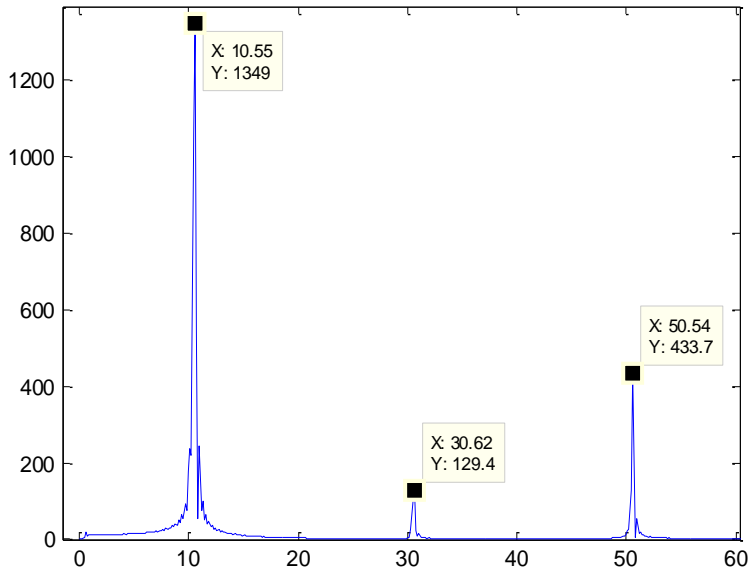

Figure 6. The results of summation of $10 \mathrm{~Hz}, 30 \mathrm{~Hz}$ and $50 \mathrm{~Hz}$ sinusoidal signal as the complex input signal using $150 \mathrm{~Hz}$ sampling rate

Table 4. The frequency detection on fix $150 \mathrm{~Hz}$

\begin{tabular}{ccc}
\multicolumn{3}{c}{ sampling rate } \\
\hline SInput Frequency & Detected frequency & Accuracy $(\%)$ \\
\hline 10 & 10.546875 & 94.53 \\
20 & 20.361328 & 98.19 \\
30 & 30.615234 & 97.95 \\
40 & 40.576172 & 98.56 \\
50 & 50.537109 & 98.93 \\
\hline
\end{tabular}

\section{CONCLUSION}

In this paper, the LCPSDC has been reviewed. The effect of the sampling rate of the algorithm to the accuracy of algorithm has been investigated. The relationship of the sampling rate and the accuracy are inversely proportional. The effect of the input frequency to the accuracy of the algorithm also has been investigated. The relationship of the input frequency and the accuracy is directly proportional where the accuracy is higher with the increasing of input frequency. The experiment has determined that LCPSDC has the ability to perform complex signal too. Further experiment will be done to the LCPSDC in future to investigate the optimum parameter for apply in biosensor signal application.

\section{ACKNOWLEDGEMENTS}

The authors would like to acknowledge the support from the Fundamental Research Grant Scheme (FRGS) under a grant number of FRGS/1/2014/SG05/UNIMAP/02/3 from the Ministry of Higher Education Malaysia.

\section{REFERENCES}

[1] B.Spangl andR.Dutter, "Analyzing short-term measurements of heart rate variability in the frequency domain using robustly estimated spectral density functions," Comput. Stat. Data Anal., vol. 56, no. 5, pp. 1188-1199, May2012.

[2] M.Ugur, S.Cekli, andC. P.Uzunoglu, "Amplitude and frequency detection of power system signals with chaotic distortions using independent component analysis," Electr. Power Syst. Res., vol. 108, pp. 43-49, Mar.2014.

[3] A. J.Barbour andR. L.Parker, "psd: Adaptive, sine multitaper power spectral density estimation for R," Comput. Geosci., vol. 63, pp. 1-8, Feb.2014.

[4] K. K.Parhi andM.Ayinala, "Low-Complexity Welch Power Spectral Density Computation," IEEE Trans. Circuits Syst. I Regul. Pap., vol. 61, no. 1, pp. 172-182, Jan.2014.

[5] L.Stanciu andC.Stanciu, "Grouped B-spline windows for power spectral density estimation," in International Symposium on Signals, Circuits and Systems ISSCS2013, 2013, no. 6, pp. 1-4.

[6] T.Schaffer, B.Hensel, C. W.Ju, andC.Jeleazcov, "Evaluation of techniques for estimating the power spectral density of RR-intervals under paced respiration conditions," J. Clin. Monit. Comput., vol. 28, no. 5, pp. 481-486, 2014.

[7] N.Balasaraswathy andR.Rajavel, "Low-complexity Power Spectral Density Estimation Power spectral density," in Artificial Intelligence and Evolutionary Algorithms in Engineering Systems, 2015, pp. 273-282. 
[8] X.Liu, C.Zhang, Z.Ji, Y.Ma, andX.Shang, "Multiple characteristics analysis of Alzheimer's electroencephalogram by power spectral density and Lempel - Ziv complexity," Cogn. Neurodyn., vol. 10, no. 2, pp. 121-133, 2016.

[9] Z.Sun, D.Tian, andX.Ning, "Parameter Estimation Technique for the SNCK Scheme Based on the SpectralCorrelation Density," Wirel. Pers. Commun., vol. 82, no. 3, pp. 1505-1529, 2015.

[10] R.Wang, J.Wang, H.Yu, X.Wei, C.Yang, andB.Deng, "Power spectral density and coherence analysis of Alzheimer 's EEG," Cogn. Neurodyn., vol. 9, no. 3, pp. 291-304, 2015.

[11] V.Radha, C.Vimala, andM.Krishnaveni, "Power Spectral Density Estimation Using Yule Walker AR Method for Tamil Speech Signal," Inf. Syst. Indian Lang., pp. 284-288, 2011.

[12] A.Nasser, A.Mansour, K. C.Yao, H.Abdallah, andH.Charara, "Spectrum sensing based on cumulative power spectral density," EURASIP J. Adv. Signal Process., 2017.

[13] G.Huang, J.Meng, D.Zhang, andX.Zhu, "Window Function for EEG Power Density Estimation and Its Application in SSVEP Based BCIs," in International Conference on Intelligent Robotics and Applications, 2011, pp. 135-144.

[14] P.Welch, "The use of fast Fourier transform for the estimation of power spectra: A method based on time averaging over short, modified periodograms," IEEE Trans. Audio Electroacoust., vol. 15, no. 2, pp. 70-73, Jun.1967.

[15] J. W.Leis, Digital Signal Processing Using MATLAB for Students and Researchers. Hoboken, NJ, USA: John Wiley \& Sons, Inc., 2011.

\section{BIOGRAPHIES OF AUTHORS}

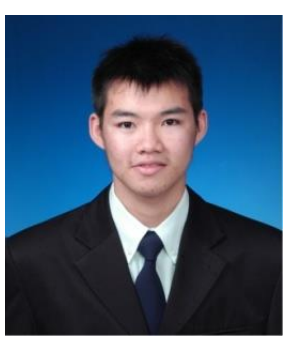

Teh Yi Jun is currently a PhD student in School of Microelectronics Engineering, Universiti Malaysia Perlis. His main research is on nano-bio sensors signal characteristics and its on-chip analysis algorithms for early diseases detection. He has his B. Eng. degree in Microelectronic engineering from Universiti Malaysia Perlis in 2014.

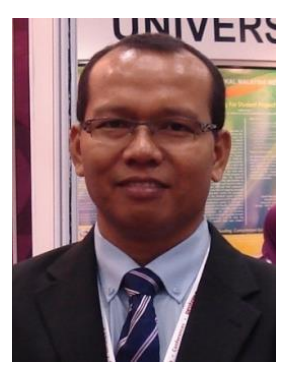

Dr. Asral Bahari is a senior lecturer at the School of Microelectronics Engineering, Universiti Malaysia Perlis (UniMAP), and was a Programme Chairperson for the Electronics Engineering Degree Programme, UniMAP, from September 2009 to Mac 2013. He has more than 15 years' experience in VLSI design in both the industry and academic sectors, and has been involved at various levels of VLSI design such as transistor modelling, digital circuit design, analogue circuit design, logic synthesis and physical place and route, architecture design and algorithm development.

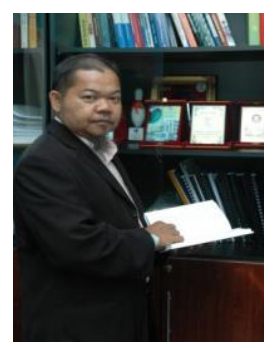

Prof. Dr. Uda Hashim is a Professor at Institute of Nano Electronic Engineering (INEE), Universiti Malaysia Perlis (UniMAP), and was a Director for the INEE, UniMAP since October 2008. He has more than 25 years experience in Semiconductor Devices, CMOS Based Sensor, Nanoelectronic and Nano Biochip in both the industry and academic sectors. 